\title{
Does our Mycobacteriome Influence COVID-19 Morbidity and Lethality?
}

\author{
Armando Acosta $^{1 *}$, Luis Fonte ${ }^{2 *}$, Maria E. Sarmiento ${ }^{1 *}$ and Mohd Nor Norazmi ${ }^{1 *}$ \\ ${ }^{1}$ School of Health Sciences, Universiti Sains Malaysia, Kelantan, Malaysia, ${ }^{2}$ Department of Parasitology, Institute of Tropical \\ Medicine "Pedro Kouri", Havana, Cuba
}

Keywords: mycobacteriome, COVID-19, bacille calmette-guérin vaccine, non-tuberculous mycobacteria, Latent TB infections

\section{OPEN ACCESS}

Edited by:

Ulrich Emil Schaible,

Research Center Borstel

(LG), Germany

Reviewed by:

Matthias Hauptmann

Research Center Borstel

(LG), Germany

*Correspondence:

Armando Acosta

armando@usm.my

Luis Fonte

luisfonte@infomed.sld.cu

Maria E. Sarmiento

mari@usm.my

Mohd Nor Norazmi

norazmimn@usm.my

Specialty section: This article was submitted to

Microbial Immunology,

a section of the journal

Frontiers in Microbiology

Received: 03 August 2020

Accepted: 14 January 2021

Published: 04 February 2021

Citation:

Acosta A, Fonte L, Sarmiento ME and Norazmi MN (2021) Does our

Mycobacteriome Influence COVID-19

Morbidity and Lethality?

Front. Microbiol. 12:589165.

doi: 10.3389/fmicb.2021.589165
COVID-19, caused by SARS-CoV-2, can be asymptomatic, manifest as mild clinical symptoms, or go to pneumonia and multi-organ failure that can be fatal. This range of clinical manifestations is a consequence of the activation of the innate and adaptive immune responses which seem to contain viral replication and lead to recovery and, in the more unfavorable sequelae, can trigger an intense inflammatory reaction, leading to serious clinical complications, and death (Wang et al., 2020).

Despite the concerns on the health care infrastructures and the high proportion of people living in poverty and overcrowded urban settings, COVID-19 in Sub-Saharan Africa (SSA) has not resulted in the anticipated huge number of confirmed cases and deaths (World Health Organization, 2020).

A combination of several factors has been put forward to explain the unexpectedly lower rates of incidence and lethality-non-extensive diagnostic testing, younger age group, host genetic background, SARS-CoV-2 mutations, higher temperature, environmentally less-favorable for viral replication, endemic infections, Bacillus Calmette-Guérin (BCG) vaccination policy, and microbiome, among others (Fonte et al., 2020; Janda et al., 2020; Khatiwada and Subedi, 2020; Mbow et al., 2020).

One of the components of the human microbiome is the mycobacteriome, composed by mycobacteria (Macovei et al., 2015). The term "mycobacteriome" was coined associated with nontuberculous mycobacteria (NTM), defined by the authors as "non-tuberculous mycobacteriome" (Macovei et al., 2015), but considering the presence of BCG decades after vaccination (Armbruster et al., 1990; Van Deutekom et al., 1996; Talbot et al., 1997) and the evidence of Mycobacterium tuberculosis (Mtb) infection in healthy individuals [latent tuberculosis infection (LTBI)] (BarriosPayán et al., 2012; Mayito et al., 2019; Mehaffy et al., 2020), we consider that the concept of mycobacteriome could be expanded including NTM, BCG, and Mtb.

Many factors influence microbiome composition, such as genetic background, environmental and socioeconomic conditions, geographical region, lifestyles, and alimentary habits, among others. BCG vaccination, the presence of LTBI and environmental NTM, in low- and medium-income countries, such as those that belong to SSA, may present a distinctive mycobacteriome compared to those of high-income countries. This distinct mycobacteriome has been suggested to influence the susceptibility to infections, allergy, and autoimmunity (Sewell et al., 2002; Obihara et al., 2006; Nemeth et al., 2019).

Recent reports suggested the possibility that BCG vaccination has a positive effect on the prevention of COVID-19 and its lethality (Escobar et al., 2020; Netea et al., 2020). The explanation of this phenomenon has been based on the "trained immunity" mechanism, induced by BCG vaccination, which has been demonstrated for different infectious diseases in children and in experimental viral infection in humans (Arts et al., 2018; Netea et al., 2020). It has been also suggested that the protective effect of BCG could be mediated by specific responses against shared 
epitopes between SARS-CoV-2 and BCG (Reche, 2020; Strongin et al., 2020; Tomita et al., 2020). However, some authors argued that the protective effect of BCG against COVID-19 is not clearly demonstrated, and after considering potential confounding factors, such as SARS-CoV-2 testing rate, the protective effect vanished (Hensel et al., 2020; Redelman-Sidi, 2020; Shivendu et al., 2020; Wassenaar et al., 2020).

The impact of the mycobacteriome on the protection against SARS-CoV-2 infection and its related disease could probably be explained beyond BCG vaccination. The potential impact of LTBI, present in one-quarter of the human population (Houben and Dodd, 2016), with high prevalence in regions where BCG immunization is implemented, should be also considered. It has been suggested that the positivity of Tuberculin Skin Test (TST) and/or Interferon Gamma Release Assays correlate better with lower incidence and lethality of COVID-19 than with BCG vaccination (Singh, 2020). However, a small study with 36 positive SARS-CoV-2 cases suggested a detrimental effect of LTBI and TB on the COVID-19 evolution (Liu et al., 2020).

LTBI triggers an increase in M1 macrophage presence, both in mice and humans, which is compatible with the activation of innate immune responses, as has been described in the context of "trained immunity" (Nemeth et al., 2019; Netea et al., 2020). The same results have also been reported with vaccine candidates based on attenuated Mtb strains in mice (Tarancón et al., 2020). It has been reported that LTBI prevents asthma and abrogates eosinophilopoiesis in an experimental model (Tarancón et al., 2019). LTBI was associated with protection against allergy in TB endemic areas (Obihara et al., 2006). The same study found that the intensity of the TST reactivity was inversely correlated with the development of allergic reactions, which suggested a role of a biased Th1-type response with downregulation of Th2 responses (Obihara et al., 2006). These studies support the opinion that LTBI, through mechanisms of innate immunity, produces

\section{REFERENCES}

Armbruster, C., Junker, W., Vetter, N., and Jaksch, G. (1990). Disseminated bacille calmette-guérin infection in an AIDS patient 30 years after BCG vaccination. J. Infect. Dis. 162:1216. doi: 10.1093/infdis/162.5.1216

Arts, R. J., Moorlag, S. J., Novakovic, B., Li, Y., Wang, S.-Y., Oosting, M., et al. (2018). BCG vaccination protects against experimental viral infection in humans through the induction of cytokines associated with trained immunity. Cell Host Microbe 23, 89-100.e5. doi: 10.1016/j.chom.2017.12.010

Barrios-Payán, J., Saqui-Salces, M., Jeyanathan, M., Alcántara-Vazquez, A., Castañon-Arreola, M., Rook, G., et al. (2012). Extrapulmonary locations of Mycobacterium tuberculosis DNA during latent infection. J. Infect. Dis. 206, 1194-1205. doi: 10.1093/infdis/jis381

Escobar, L. E., Molina-Cruz, A., and Barillas-Mury, C. (2020). BCG vaccine protection from severe coronavirus disease 2019 (COVID-19). Proc. Natl. Acad. Sci. U.S.A. 117, 17720-17726. doi: 10.1073/pnas.2008410117

Fonte, L., Acosta, A., Sarmiento, M. E., Ginori, M., García, G., and Norazmi, M. N. (2020). COVID-19 lethality in Sub-Saharan Africa and helminth immune modulation. Front. Immunol. 11:574910. doi: 10.3389/fimmu.2020.574910

Hensel, J., McGrail, D. J., McAndrews, K. M., Dowlatshahi, D., LeBleu, V. S., and Kalluri, R. (2020). Exercising caution in correlating COVID-19 incidence and mortality rates with BCG vaccination policies due to variable rates of SARS CoV-2 testing. medRxiv. doi: 10.1101/2020.04.08.20056051 a bystander effect on acquired immune responses, which could generate a protective environment against heterologous microorganisms, including SARS-CoV-2.

Other members of the mycobacteriome with potential influence on COVID-19 severity are NTM, which, due to their worldwide distribution, are also present in areas covered by BCG vaccination. NTM have been associated with the production of a regulatory environment mediated by transforming growth factor- $\beta$ (TGF- $\beta$ ) and interleukin-10 (IL-10), induction of $\mathrm{T}$ regulatory cells, and inhibition of neutrophil infiltration (Zuany-Amorim et al., 2002a,b). Such an environment could provide protection against the severe forms of COVID-19, inhibiting the uncontrolled inflammation resulting in the so-called "cytokine storm."

In summary, the lower incidence and lethality rates of COVID-19 registered in SSA countries could be a consequence, among other factors, of a blend of different immunological mechanisms, some of them induced by members of the mycobacteriome: BCG and LTBI affecting SARS-CoV-2 multiplication through trained immunity and cross-reactive immune responses, and NTM limiting the pathological inflammation triggered by the host immune response against the virus, through the induction of a regulatory environment.

\section{AUTHOR CONTRIBUTIONS}

All authors listed have made a substantial, direct and intellectual contribution to the work, and approved it for publication.

\section{FUNDING}

The authors acknowledge funding from the LRGS Grant 203.PPSK.67212002 (Ministry of Education, Malaysia).

Houben, R. M., and Dodd, P. J. (2016). The global burden of latent tuberculosis infection: a re-estimation using mathematical modelling. PLoS Med. 13:e1002152. doi: 10.1371/journal.pmed.1002152

Janda, L., Mihalčin, M., and Štastná, M. (2020). Is a healthy microbiome responsible for lower mortality in COVID-19? Biologia. doi: 10.2478/s11756-020-00614-8. [Epub ahead of print].

Khatiwada, S., and Subedi, A. (2020). Lung microbiome and coronavirus disease 2019 (COVID-19): possible link and implications. Hum Microb J. 17:100073. doi: 10.1016/j.humic.2020.100073

Liu, Y., Bi, L., Chen, Y., Wang, Y., Fleming, J., Yu, Y., et al. (2020). Active or latent tuberculosis increases susceptibility to COVID-19 and disease severity. medRxiv. doi: 10.1101/2020.03.10.20033795

Macovei, L., McCafferty, J., Chen, T., Teles, F., Hasturk, H., Paster, B. J., et al. (2015). The hidden 'mycobacteriome'of the human healthy oral cavity and upper respiratory tract. J. Oral Microbiol. 7:26094. doi: 10.3402/jom.v7.26094

Mayito, J., Andia, I., Belay, M., Jolliffe, D. A., Kateete, D. P., Reece, S. T., et al. (2019). Anatomic and cellular niches for Mycobacterium tuberculosis in latent tuberculosis infection. J. Infect. Dis. 219, 685-694. doi: 10.1093/infdis/jiy579

Mbow, M., Lell, B., Jochems, S. P., Cisse, B., Mboup, S., Dewals, B. G., et al. (2020). COVID-19 in Africa: dampening the storm? Science 369, 624-626. doi: 10.1126/science.abd3902

Mehaffy, C., Kruh-Garcia, N. A., Graham, B., Jarlsberg, L. G., Willyerd, C. E., Borisov, A., et al. (2020). Identification of Mycobacterium tuberculosis peptides 
in serum extracellular vesicles from persons with latent tuberculosis infection. J. Clin. Microbiol. 58, e00393-20. doi: 10.1128/JCM.00393-20

Nemeth, J., Olson, G. S., Rothchild, A., Jahn, A., Mai, D., Duffy, F., et al. (2019). Latent Mycobacterium tuberculosis infection provides protection for the host by changing the activation state of the innate immune system. bioRxiv 561126 . doi: $10.1101 / 561126$

Netea, M. G., Giamarellos-Bourboulis, E. J., Domínguez-Andrés, J., Curtis, N., van Crevel, R., van de Veerdonk, F. L., et al. (2020). Trained immunity: a tool for reducing susceptibility and severity of SARS-CoV-2 infection. Cell 181, 969-977. doi: 10.1016/j.cell.2020.04.042

Obihara, C. C., Kimpen, J. L., Gie, R. P., van Lill, S. W., Hoekstra, M. O., Marais, B. J., et al. (2006). Mycobacterium tuberculosis infection may protect against allergy in a tuberculosis endemic area. Clin. Exp. Allergy 36, 70-76. doi: $10.1111 / j .1365-2222.2005 .02408 . x$

Reche, P. A. (2020). Potential cross-reactive immunity to SARS-CoV-2 from common human pathogens and vaccines. Front. Immunol. 11:586984. doi: $10.3389 /$ fimmu.2020.586984

Redelman-Sidi, G. (2020). Could BCG be used to protect against COVID-19? Nat. Rev. Urol. 17, 316-317. doi: 10.1038/s41585-0200325-9

Sewell, D. L., Reinke, E. K., Hogan, L. H., Sandor, M., and Fabry, Z. (2002). Immunoregulation of CNS autoimmunity by helminth and mycobacterial infections. Immunol. Lett. 82, 101-110. doi: 10.1016/S0165-2478(02) 00025-1

Shivendu, S., Chakraborty, S., Onuchowska, A., Patidar, A., and Srivastava, A. (2020). Is there evidence that BCG vaccination has non-specific protective effects for COVID-19 infections or is it an illusion created by lack of testing? SSRN 3579847. doi: 10.2139/ssrn.3579847

Singh, S. (2020). BCG vaccines may not reduce COVID-19 mortality rates. medRxiv. doi: 10.1101/2020.04.11.20062232

Strongin, A. Y., Sloutsky, A., and Cieplak, P. (2020). Note on the potential BCG vaccination-COVID-19 molecular link. Coronaviruses 1, 4-6. doi: 10.2174/26667967019992006290 03417

Talbot, E. A., Perkins, M. D., Silva, S. F. M., and Frothingham, R. (1997). Disseminated bacille calmette-guerin disease after vaccination: case report and review. Clin. Infect. Dis. 24, 1139-1146. doi: 10.1086/ 513642

Tarancón, R., Domínguez-Andrés, J., Uranga, S., Ferreira, A. V., Groh, L. A., Domenech, M., et al. (2020). New live attenuated tuberculosis vaccine MTBVAC induces trained immunity and confers protection against experimental lethal pneumonia. PLoS Pathog 16:e1008404. doi: 10.1371/journal.ppat.1008404
Tarancón, R., Uranga, S., Martín, C., and Aguiló, N. (2019). Mycobacterium tuberculosis infection prevents asthma and abrogates eosinophilopoiesis in an experimental model. Allergy 74, 2512-2514. doi: 10.1111/all.13923

Tomita, Y., Sato, R., Ikeda, T., and Sakagami, T. (2020). BCG vaccine may generate cross-reactive T cells against SARS-CoV-2: in silico analyses and a hypothesis. Vaccine 38, 6352-6356. doi: 10.1016/j.vaccine.2020.08.045

Van Deutekom, H., Smulders, Y., Roozendaal, K., and Van Soolingen, D. (1996). Bacille calmette-cuerin (BCG) meningitis in an AIDS patient 12 years after vaccination with BCG. Clin. Infect. Dis. 22, 870-871. doi: $10.1093 /$ clinids/22.5.870

Wang, Y., Wang, Y., Chen, Y., and Qin, Q. (2020). Unique epidemiological and clinical features of the emerging 2019 novel coronavirus pneumonia (COVID-19) implicate special control measures. J. Med. Virol. 92, 568-576. doi: 10.1002/jmv.25748

Wassenaar, T. M., Buzard, G., and Newman, D. (2020). BCG vaccination early in life does not improve COVID-19 outcome of elderly populations, based on nationally reported data. Lett. Appl. Microbiol. 71, 498-505. doi: $10.1111 /$ lam. 13365

World Health Organization (2020). Coronavirus disease (COVID-19) Weekly Epidemiological Update and Weekly Operational Update. Geneva: World Health Organization (2020). Available online at: https://www.who.int/ emergencies/diseases/novel-coronavirus-2019/situation-reports (accessed October 11, 2020).

Zuany-Amorim, C., Manlius, C., Trifilieff, A., Brunet, L. R., Rook, G., Bowen, G., et al. (2002b). Long-term protective and antigen-specific effect of heat-killed Mycobacterium vaccae in a murine model of allergic pulmonary inflammation. J Immunol. 169, 1492-1499. doi: 10.4049/jimmunol.169.3.1492

Zuany-Amorim, C., Sawicka, E., Manlius, C., Le Moine, A., Brunet, L. R., Kemeny, D. M., et al. (2002a). Suppression of airway eosinophilia by killed Mycobacterium vaccae-induced allergen-specific regulatory T-cells. Nat. Med. 8, 625-629. doi: $10.1038 / \mathrm{nm} 0602-625$

Conflict of Interest: The authors declare that the research was conducted in the absence of any commercial or financial relationships that could be construed as a potential conflict of interest.

Copyright $\odot 2021$ Acosta, Fonte, Sarmiento and Norazmi. This is an open-access article distributed under the terms of the Creative Commons Attribution License (CC $B Y)$. The use, distribution or reproduction in other forums is permitted, provided the original author(s) and the copyright owner(s) are credited and that the original publication in this journal is cited, in accordance with accepted academic practice. No use, distribution or reproduction is permitted which does not comply with these terms. 